\title{
Investigation of Electron Extraction from a Microwave Discharge Neutralizer for a Miniature Ion Propulsion System
}

\author{
By Yoshinori TAKAO, ${ }^{1)}$ Hiroyuki KoIZUMI, ${ }^{2)}$ Yusuke KASAGI ${ }^{2)}$ and Kimiya KomURASAKI ${ }^{2)}$ \\ ${ }^{1)}$ Division of Systems Research, Yokohama National University, Yokohama, Japan \\ ${ }^{2)}$ Department of Aeronautics and Astronautics, The University of Tokyo, Tokyo, Japan
}

(Received July 16th, 2015)

\begin{abstract}
To investigate electron extraction through the orifices of a microwave discharge neutralizer, three-dimensional particle simulations have been conducted. The numerical model is composed of a particle-in-cell simulation with a Monte Carlo collision algorithm for the kinetics of charged particles, a finite-difference time-domain method for the electromagnetic fields of $4.2-\mathrm{GHz}$ microwaves, and a finite element analysis for the magnetostatic fields of permanent magnets. The distribution of the current density on the orifice plate obtained from the numerical model is in a reasonable agreement with the measurement result in an experiment. Moreover, the numerical results have indicated that the electrostatic field of the plasma has a dominant influence on the electron extraction although the electrostatic field produces the opposite force of extraction from the bulk plasma toward the orifice plate. The combination of the sheath potential barrier and the magnetostatic field yields the electron trajectories of extraction.
\end{abstract}

Key Words: Electron Extraction, Neutralizer, Microwave, ECR, PIC-MCC

\section{Nomenclature}

$\begin{array}{lll}\boldsymbol{B}_{\text {st }} & : \text { magnetostatic field } \\ \boldsymbol{E} & : \text { electric field } \\ \boldsymbol{j} & : \text { current density } \\ m & : \text { mass } \\ n & : \text { number density } \\ p & : \text { pressure } \\ P & : \text { power } \\ q & : \text { charge } \\ t & : \text { time } \\ T & : \text { temperature } \\ \boldsymbol{v} & : \text { velocity } \\ \boldsymbol{x} & : \text { position } \\ \varepsilon_{0} & : \text { vacuum permittivity } \\ \mathcal{E}_{\mathrm{r}} & : \text { relative permittivity } \\ \mu_{0} & : \text { vacuum permeability } \\ \rho & : \text { charge density } \\ \phi & : \text { electrostatic potential } \\ \omega & : \text { microwave angular frequency }\end{array}$

Subscripts

$0 \quad$ : initial

abs : absorption

e : electron

EM : electromagnetic

ES : electrostatic

i : ion

$\mathrm{p} \quad$ : plasma

\section{Introduction}

Low costs and short development periods have enabled even small companies and universities to launch and operate microspacecraft, which brings a good opportunity for education and a new business. However, most microspacecraft do not have any propulsion systems, and thus they rely on passive control systems, such as gravity gradient stabilization and magnetic torquers. If a microspacecraft had microthrusters mounted on it, the microspacecraft could be controlled more effectively and inserted into a specific orbit by itself. To realize a microthruster installable to microspacecraft, high specific impulses, high-thrust efficiencies and low-power consumptions are required in addition to small size and light weight because of the limited power generation and propellant storage. ${ }^{1)}$

To satisfy such requirements, a miniature ion propulsion system (MIPS) was developed by the University of Tokyo. ${ }^{2-4)}$ The MIPS employs electron cyclotron resonance (ECR) discharges with ring-shaped permanent magnets for its ion source and neutralizer. ${ }^{5-7)}$ The FM specifications of the MIPS are evaluated at the weight of $8.1-8.6 \mathrm{~kg}$ (dry: $7.2 \mathrm{~kg}$ ), volume of $34 \times 26 \times 16 \mathrm{~cm}^{3}$, power consumption of $27-34 \mathrm{~W}$ and thrust of $210-300 \mu \mathrm{N}$ with specific impulse of $740-1100 \mathrm{~s}$. The MIPS was installed on a $50 \mathrm{~kg}$-class spacecraft, HODOYOSHI-4, which was launched on June 19, 2014, and was operated successfully in space on October 28.

Although the MIPS has already been utilized in space, there are still some physical phenomena to be clarified especially in the mechanism of electron extraction from its neutralizer. Owing to the small size of the MIPS, the neutralizer operates with the identical discharge chamber, the same level of microwave power, and a half gas flow rate of the ion source, ${ }^{3)}$ which indicates that the MIPS neutralizer consumes the resources (space, power, and propellant) more significantly than conventional ion propulsion systems. One of the reasons for a poor performance is considered to be a magnetic confinement of 


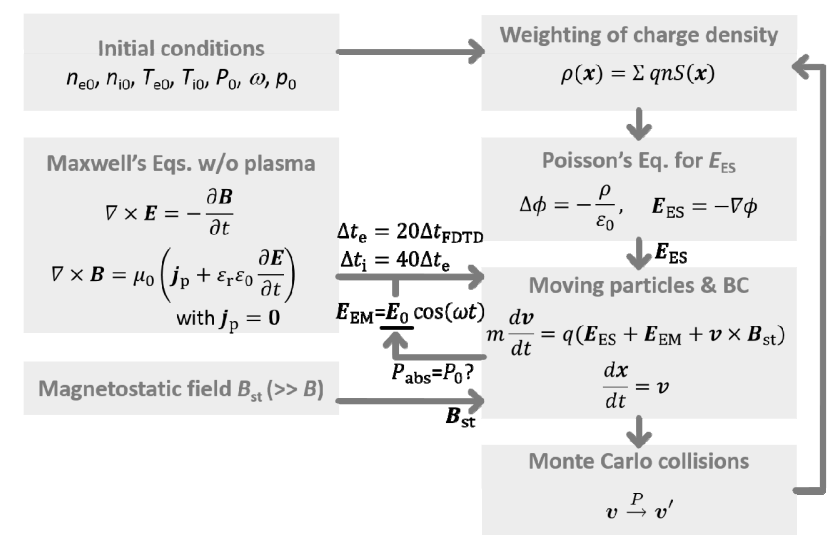

Fig. 1. Flow chart for the numerical model presented. First, we set initial conditions and then we solve Maxwell's equations by FDTD for the electromagnetic fields of microwaves to obtain a steady state without plasma. We use the amplitude of the electric fields of microwaves $\boldsymbol{E}_{0}$ at steady state. Second, electrostatic PIC-MCC calculations are performed by using the electrostatic field $\boldsymbol{E}_{\mathrm{ES}}$, the time-varying electric field of microwaves $\boldsymbol{E}_{\mathrm{EM}}$, and the magnetostatic fields $\boldsymbol{B}$ st, which is determined with ANSYS software. In the simulation, the power absorbed in the plasma is used as an input parameter.

electrons inside the plasma source. Hence, it would be important to investigate the process through which electrons are extracted from the plasma source with applied magnetic fields.

In order to elucidate the mechanism for a better performance of the neutralizer, numerical simulations could be a powerful tool to compensate for lack of information obtained from experiments. Hence, we have developed a three-dimensional numerical model, which consists of a particle-in-cell simulation with a Monte Carlo collision algorithm (PIC-MCC) for the kinetics of charged particles, a finite-difference time-domain (FDTD) algorithm for the electromagnetic fields of microwaves, and a finite element analysis for the magnetostatic fields of permanent magnets.

In the present work, to investigate electron extraction from the microwave discharge neutralizer of the MIPS, we have conducted PIC-MCC simulations. In the next section, we briefly describe our numerical model, followed by the experimental procedure for validation. The characteristics of the microwave discharge plasma and its current distribution are indicated and compared with the experimental results in section 4 . The validated numerical model would be useful to design better performance neutralizers. We have also investigated what causes electron extraction from the neutralizer, and have found that the self-generated electrostatic field of the plasma is the most important factor. Finally, conclusions are drawn of this paper in section 5 .

\section{Numerical Model}

The three-dimensional PIC-MCC calculations based on the previous papers ${ }^{8-11)}$ were carried out under the following assumptions. (i) Only singly-ionized xenon and electrons are treated as particles, where the reactions taken into account are elastic scattering, excitation, and ionization for electrons, and elastic scattering and charge exchange for ions. The cross-section data set is the same as that employed in 9). (ii)
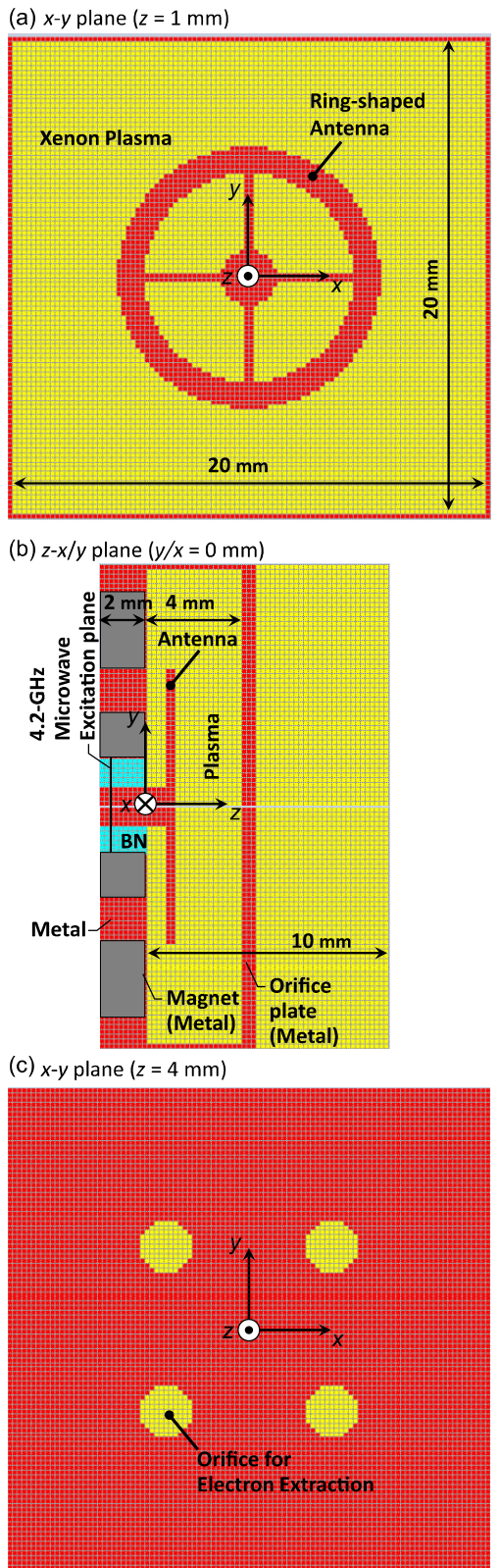

Fig. 2. Computational area and grids for calculations of the microwave discharge neutralizer with extraction of electrons: (a) $x-y$ plane $(z=1 \mathrm{~mm})$ at the antenna, (b) $z-x / y$ plane $(y / x=0 \mathrm{~mm})$, and (c) $x-y$ plane $(z=4 \mathrm{~mm})$ at the orifice plate.

The magnetic fields of microwaves are neglected compared with the magnetostatic fields of the permanent magnets. (iii) The effect of the plasma current is not taken into account owing to the low power of microwaves in this study.

Figure 1 shows the flow chart for the numerical model employed. First, we set initial conditions and then we solve Maxwell's equations by FDTD for the electromagnetic fields of microwaves with a time increment $\Delta t_{\mathrm{EM}}=2.98 \times 10^{-13} \mathrm{~s}$ $(1 / 800$ of a microwave cycle for $4.2 \mathrm{GHz})$ to obtain a steady state solution without plasma. We use the amplitude of the electric fields of microwaves $\boldsymbol{E}_{0}$ at steady state. Second, electrostatic PIC-MCC calculations are performed with a time step $\Delta t_{\mathrm{e}}=5.95 \times 10^{-12} \mathrm{~s}(1 / 40$ of a microwave cycle $)$ by using the electrostatic field $\boldsymbol{E}_{\mathrm{ES}}$ of the plasma, the time-varying electric field of microwaves $\boldsymbol{E}_{\mathrm{EM}}=\boldsymbol{E}_{0} \cos (\omega t)$, and the 
(a)

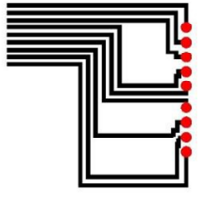

(b)
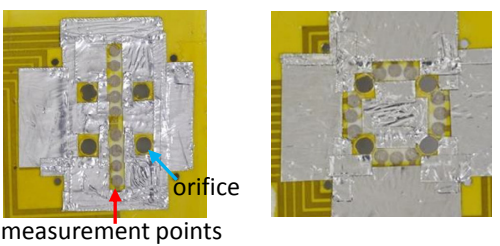

Fig. 3. Schematics of the circuits (top) and photograph images (bottom) of the current probes. The closed circles in red represent the measurement points of the current. The measurement points are set at (a) $r=1,2,3,4$, 5, 6, 7, 8, $9 \mathrm{~mm}$, (b) $r=5 \mathrm{~mm}$, and (c) $r=7.5 \mathrm{~mm}$.

magnetostatic fields $\boldsymbol{B}_{\text {st }}$ produced by the permanent magnets, which is determined with ANSYS software. Here, in order to speed up the simulation, the motion of ions is updated with a time step $\Delta t_{\mathrm{i}}=2.38 \times 10^{-10} \mathrm{~s}$ (one microwave cycle) by neglecting $\boldsymbol{E}_{\mathrm{EM}}$ and using the time-averaged $\boldsymbol{E}_{\mathrm{ES}}$ over one microwave cycle because the frequency of $4.2 \mathrm{GHz}$ is much higher than the ion plasma frequency. In the simulation, the power absorbed in the plasma $P_{\text {abs }}$ is used as an input parameter, where $P_{\text {abs }}$ is obtained by calculating the change in kinetic energy of electrons and ions before and after the calculation of the equation of motion. ${ }^{12)}$ Last, we rescale the amplitude $\boldsymbol{E}_{0}$ to yield the specified power absorbed in the plasma $P_{0}$. For example, if the obtained power $P_{\text {abs }}$ is below the specified power $P_{0}$, then we increase the amplitude of the electric field $\boldsymbol{E}_{0}$. The above procedure is iterated until the steady stage solution is obtained.

Figure 2 shows the computational domain and dimensions for electron extraction in the present study. The Cartesian coordinate system is employed and its origin is set on the center of the ring-shaped antenna at the interface between the metal wall and the plasma in the $z$ direction. The lengths in the $x, y$, and $z$ directions are 20,20 , and $10 \mathrm{~mm}$, respectively, including the region for the investigation of electron extraction. We place a $0.6-\mathrm{mm}$ thick orifice plate at $z=4 \mathrm{~mm}$, where four orifices are used in this work. As boundary conditions, the potential on all the metal area is set at zero and we set $20 \mathrm{~V}$ at $z=10 \mathrm{~mm}$ for electron extraction. All electrons and ions disappear at the walls, the antenna, and the other boundaries, where no reflection or charge accumulation are assumed.

\section{Experimental Procedure}

To validate our numerical model, the current distribution on the orifice plate was measured in the experiment and its result was compared with the numerical result. Figure 3 shows schematics of the circuits and photograph images of the current probes for the measurement. The closed circles in red represent the measurement points of the current, where the diameter is all $1.5 \mathrm{~mm}$. In Fig. 3(a), measurement points are arrayed on the $x / y$ (a)

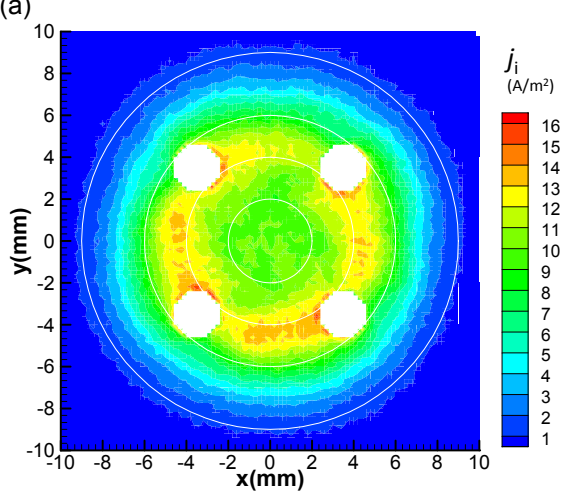

(b)

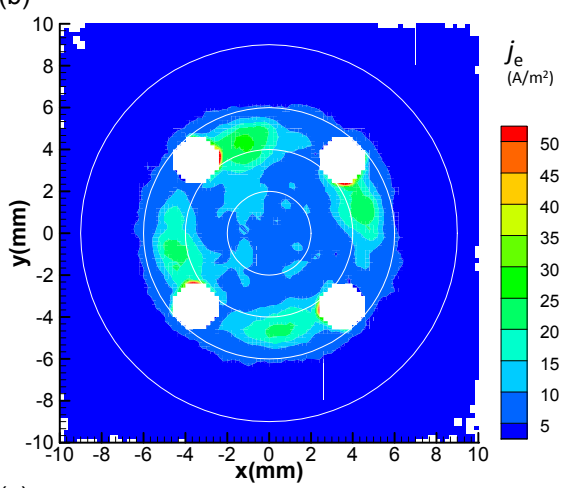

(c)

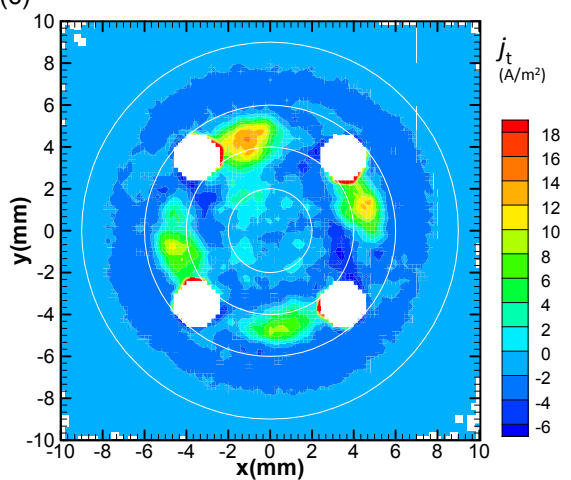

Fig. 4. Two-dimensional distributions of the (a) ion, (b) electron, and (c) total current densities flowing into the orifice plate $(z=4 \mathrm{~mm})$, together with circles in white representing the boundaries of the ring-shaped permanent magnets. Note that the contour plots are not shown inside the four orifice regions.

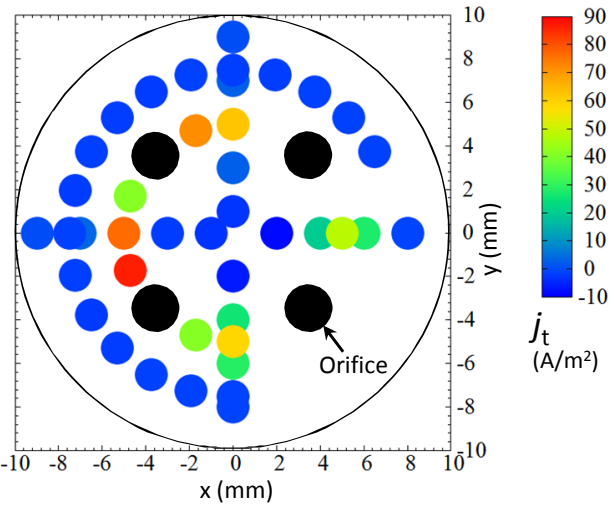

Fig. 5. Two-dimensional distributions of the total current densities flowing into the current probes in the experiment, where the input power of microwaves is $0.5 \mathrm{~W}$ and the xenon gas flow rate of $10.5 \mu \mathrm{g} / \mathrm{s}$. 
axis, where the points are set at 1, 2, 3, 4, 5, 6, 7, 8, $9 \mathrm{~mm}$ from the center of the orifice plate. There are 12 measurement points at the radius of $5 \mathrm{~mm}$ in Fig. 3(b) and 13 points at the radius of $7.5 \mathrm{~mm}$ in Fig. 3(c).

The current measurement was conducted in the vacuum chamber at the pressure of less than $1 \times 10^{-3} \mathrm{~Pa}\left(7.5 \times 10^{-6}\right.$ Torr $)$ after the orifice plate with the current probe was mounted on the microwave discharge neutralizer. The voltage of the current probes was set at $0 \mathrm{~V}$ to prevent the probe from sputtering of the plasma and to reduce the perturbation of the plasma discharge. However, setting the voltage at $0 \mathrm{~V}$ causes us to measure the total current, i.e., the sum of the ion and electron currents and thus we cannot measure electron and ion currents separately in the experiment. At the current measurement, we set the neutralizer at $0 \mathrm{~V}$ and placed an electron-collector plate with the positive potential of $20 \mathrm{~V}$ downstream of the neutralizer to extract electrons. The measurement was conducted at a xenon gas mass flow rate of $10.5 \mu \mathrm{g} / \mathrm{s}$ and the microwave input power of $0.5 \mathrm{~W}$, which is similar to the numerical conditions.

\section{Results and Discussion}

A xenon plasma discharge was calculated for the microwave discharge neutralizer under the base case condition, where the xenon gas pressure is $1 \mathrm{mTorr}$, the microwave frequency is $4.2 \mathrm{GHz}$, and the absorbed power is $0.3 \mathrm{~W}$. The initial densities of both electrons and ions are set at $1.0 \times 10^{16} \mathrm{~m}^{-3}$ and are distributed uniformly in the simulation area. The initial electron and ion temperatures are $2.0 \mathrm{eV}$ and $0.05 \mathrm{eV}$, respectively. The macroscopic parameters, such as the electron density and electron temperature were determined by averaging over 50000 microwave cycles $(11.9 \mu \mathrm{s})$ after the steady state was reached.

The peak plasma density is located in the ECR layer on the right side of the antenna, where the maximal value of the electron density is $1.6 \times 10^{17} \mathrm{~m}^{-3}$, and their distributions spread along the magnetic field lines, producing the ring-shaped profile of the plasma density. This result indicates that the plasma is well confined because of the mirror magnetic fields. The distribution of the electron temperature is almost the same as that of the plasma density, where the peak electron temperature obtained is $16 \mathrm{eV}$. The electron temperature in front of the ring antenna is much larger than the ionization energy of xenon. The details of the other results are described in our previous paper. ${ }^{9)}$

\subsection{Comparison between simulation and experiment}

Figure 4 shows two-dimensional distributions of the ion, electron, and total current densities flowing into the orifice plate. Note that the contour plots are not shown in the four orifice regions. As shown in the figure, the distribution of electron current is localized at around $r=5 \mathrm{~mm}$ (the position of the ring-shaped antenna), where the current density in the clockwise direction with respect to the orifice is higher than that in the anti-clockwise direction. The similar tendency can also be seen in the distribution of the ion current density except that the ion current density has a broader distribution. Consequently, the distribution of the total current density (the sum of the ion and electron current densities) displays the positive values around the position of the ring-shaped antenna and the negative values

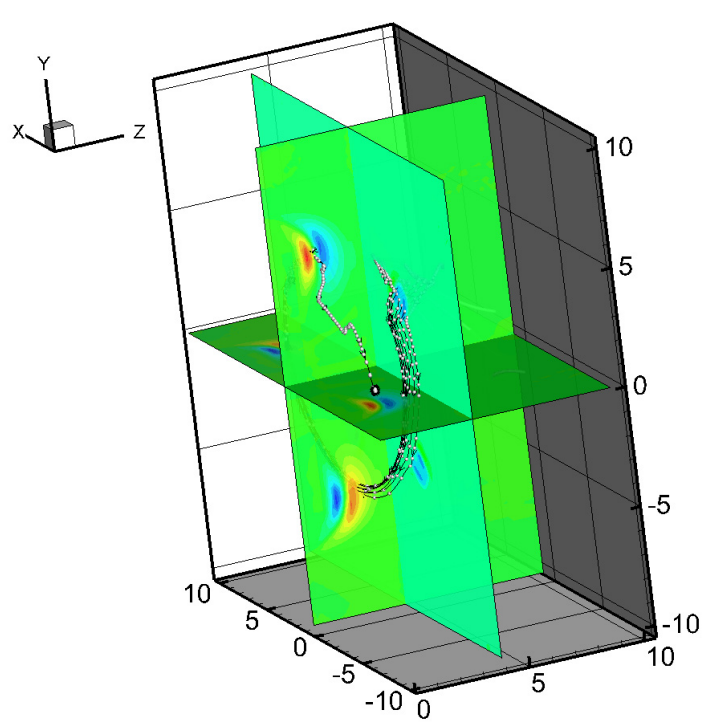

Fig. 6. Cross-sectional views of the time-averaged electron current density and a few example of its streamlines.

inside and outside it.

Figure 5 shows the two-dimensional distributions of the total current densities flowing into the current probes obtained in the experiment. As shown in the figure, the similar tendency can be seen: the peak current density is obtained at around $r=5 \mathrm{~mm}$, where the electron current is dominant over the ion current, and the current density in the clockwise direction with respect to the orifice is higher than that in the anti-clockwise direction. Even though the current density taken from the experiment is about five times larger than that obtained in the simulation, the distribution of the current density is quite similar with each other.

\subsection{Analysis of electron extraction}

To investigate the mechanism of electron extraction through the orifices of the neutralizer, we have obtained the time-averaged electron current streamlines as shown in Fig. 6, where the distributions of electron current density are also plotted as cross-sectional views. The figure clearly indicates the grad-B and/or curvature drift of electrons due to the magnetostatic field of the permanent magnets. Although the circulation of the electrons can be explained by the drift motion, the mechanism of electron motion in the $z$ direction cannot be fully understood unless only the time-averaged electron motion is focused on.

As indicated in Fig. 1 of the flow chart, the electrons are moved by the sum of the electrostatic electric field $\boldsymbol{E}_{\mathrm{ES}}$, the microwave electromagnetic field $\boldsymbol{E}_{\mathrm{EM}}$, and the magnetostatic field of the permanent magnets $\boldsymbol{B}_{\text {st. }}$. Although electron-neutral collisions also have an influence on the electron motion, the effect would be relatively small because of few collisions at the low pressure of $1 \mathrm{mTorr}$ in the small discharge chamber. To investigate what effect plays a dominant role to extract electrons from the confined plasma source through the orifice, we have conducted the PIC-MCC calculations with deleting some effects artificially after the steady state solution is obtained. Figure 7 shows some typical examples of an electron trajectory under the 

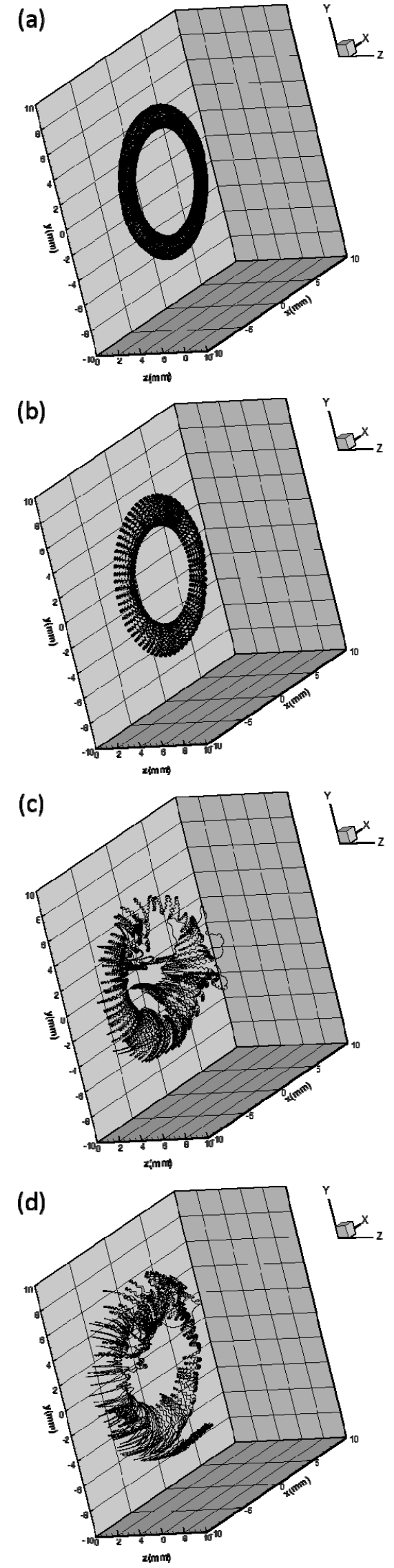

Fig. 7. Typical examples of an electron trajectory under the effect of (a) $\boldsymbol{B}_{\mathrm{st}}$ only; (b) $\boldsymbol{B}_{\mathrm{st}}$ and $\boldsymbol{E}_{\mathrm{EM}}$; (c) $\boldsymbol{B}_{\mathrm{st}}$ and $\boldsymbol{E}_{\mathrm{ES}}$; and (d) $\boldsymbol{B}_{\mathrm{st}}, \boldsymbol{E}_{\mathrm{EM}}$ and $\boldsymbol{E}_{\mathrm{ES}}$ with MCC.
Table 1. The number of electrons extracted from the plasma source through the orifice out of 100 samples chosen at random for a time span of 2,000 microwave cycles (476 ns).

\begin{tabular}{|c|c|c|c|c|}
\hline & $\boldsymbol{B}_{\mathrm{st}}$ & $\boldsymbol{B}_{\mathrm{st}}+\boldsymbol{E}_{\mathrm{EM}}$ & $\boldsymbol{B}_{\mathrm{st}}+\boldsymbol{E}_{\mathrm{ES}}$ & $\boldsymbol{B}_{\mathrm{st}}+\boldsymbol{E}_{\mathrm{EM}}+\boldsymbol{E}_{\mathrm{ES}}$ \\
\hline W/o MCC & 0 & 0 & 31 & 24 \\
\hline With MCC & 0 & 0 & 25 & 23 \\
\hline
\end{tabular}

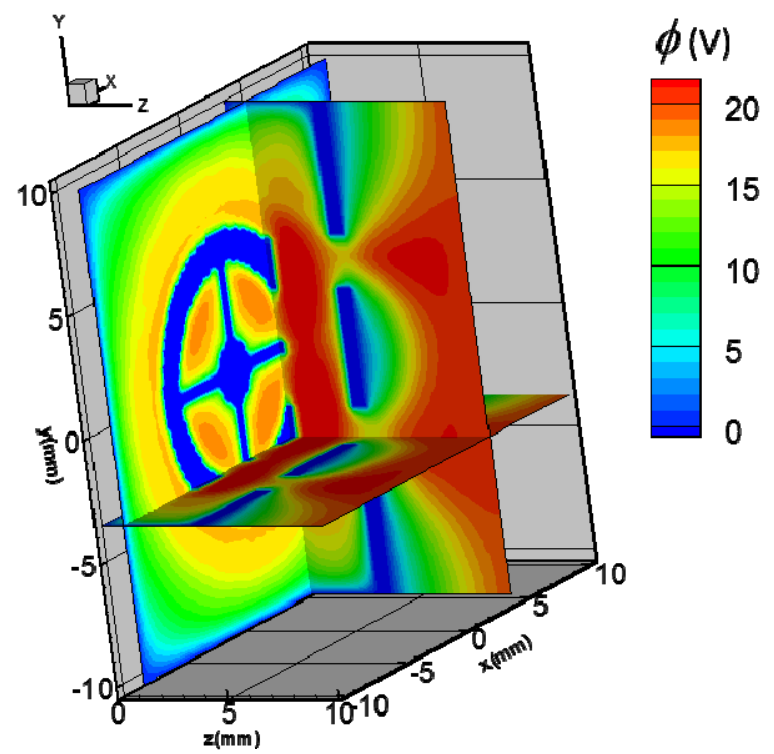

Fig. 8. Three-dimensional distribution of the time-averaged plasma potential $\phi$. The slices of contour plots are displayed at $x=3.6 \mathrm{~mm}, y=$ $-3.6 \mathrm{~mm}$, and $z=1.0 \mathrm{~mm}$.

effect of (a) $\boldsymbol{B}_{\mathrm{st}}$ only; (b) $\boldsymbol{B}_{\mathrm{st}}$ and $\boldsymbol{E}_{\mathrm{EM}}$; (c) $\boldsymbol{B}_{\mathrm{st}}$ and $\boldsymbol{E}_{\mathrm{ES}}$; and (d) $\boldsymbol{B}_{\mathrm{st}}$, $\boldsymbol{E}_{\mathrm{EM}}$ and $\boldsymbol{E}_{\mathrm{ES}}$ with collisions. The magnetostatic field $\boldsymbol{B}_{\mathrm{st}}$ causes only the mirror confinement and grad-B and/or curvature drift, so that the electron trajectory shows just a circulation. Moreover, the addition of $\boldsymbol{E}_{\mathrm{EM}}$ gives only a slight change. However, the addition of $\boldsymbol{E}_{\mathrm{ES}}$ clearly has a strong effect on the electron trajectory and the electron is extracted from the orifice. Table 1 summarizes the number of electrons extracted from the orifice out of 100 samples chosen at random for a time span of 2,000 microwave cycles ( $476 \mathrm{~ns}$ ), which indicates that electrons are not extracted unless the effect of $\boldsymbol{E}_{\mathrm{ES}}$ is taken into account in the calculations.

Figure 8 shows the three-dimensional distribution of the time-averaged plasma potential $\phi$, which is used to derive $\boldsymbol{E}_{\mathrm{ES}}$. The plasma potential of the bulk plasma is obviously higher than that at the orifice plate, which indicates that electrons have to overcome the potential barrier as well as to escape from the magnetic confinement in order to be extracted through the orifice. Although $\boldsymbol{E}_{\mathrm{ES}}$ is required for electron extraction, the distribution of $\boldsymbol{E}_{\mathrm{ES}}$ does not show a clear reason for extraction from the bulk plasma into space through the orifice.

Figure 9 shows a trajectory of the electron extracted through the orifice from the plasma source, together with the magnetostatic field lines and the distribution of the magnetic field strength. The electron moves along the magnetic field line and travels back and forth as a result of the confinement due to the mirror magnetic fields. During this motion, the trajectory of the electron moves from one of the magnetic field lines to 
(a)

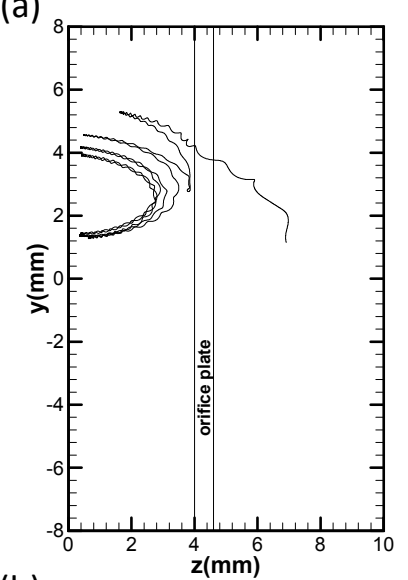

(b)

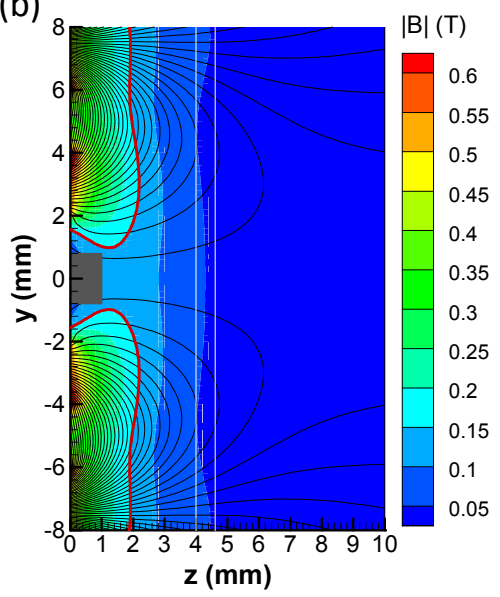

Fig. 9. (a) Typical example of a trajectory of the electron extracted through the orifice from the plasma source, together with (b) the magnetostatic field lines and the distribution of the magnetic field

another line when the electron is reflected by the potential barrier in the sheath. The trajectory is then going outward gradually, and consequently the electron is extracted through the orifice. This indirect extraction mechanism would result in a poor performance of the neutralizer.

Finally, the electron current extracted from the orifice was calculated to be $1 \mathrm{~mA}$, while the electron current lost to the wall was $4 \mathrm{~mA}$. This result shows that $20 \%$ of electrons produced in the discharge chamber are extracted from the orifice as electron beams for a neutralizer.

\section{Conclusions}

In the present work, we have conducted three-dimensional PIC-MCC simulations to investigate the electron extraction from the microwave discharge neutralizer. The PIC-MCC model includes an FDTD for the electromagnetic fields of 4.2-GHz microwaves and an FEM analysis for the magnetostatic fields of permanent magnets. The PIC-MCC results have been compared with the results obtained in the experiment to validate our model, where the distribution of the current density is quite similar with each other. To investigate what causes electron extraction from the orifices, we have conducted simulations with deleting some effects artificially. As a result, electrons are not extracted unless the effect of $\boldsymbol{E}_{\mathrm{ES}}$ is taken into account in the PIC calculation, and thus the electron extraction is mainly attributed to the self-generated electrostatic field of the plasma, even though $\boldsymbol{E}_{\mathrm{ES}}$ gives the opposite force of electron extraction from the bulk plasma toward the orifice plate. Bouncing electrons at the sheath results in the transition of electron trajectories confined by the magnetic field. The transition leads to the electron extraction. Finally, $20 \%$ of electrons produced in the discharge chamber are found to be extracted from the orifice.

\section{Acknowledgments}

This work was financially supported in part by a Grant-in-Aid for Scientific Research (B) (Grant No. 25289304) from the Japan Society for the Promotion of Science. Part of the computer simulations in the present study was performed on the KDK computer system at the Research Institute for Sustainable Humanosphere, Kyoto University.

\section{References}

1) Micci, M. M. and Ketsdever, A. D.: Micropropulsion for Small Spacecraft, American Institute of Aeronautics and Astronautics, Reston, 2000.

2) Koizumi, H., Komurasaki, K., Aoyama, J., and Yamaguchi, K.: Engineering Model of the Miniature Ion Propulsion System for the Nano-satellite: HODOYOSHI-4, Trans. JSASS Aerospace Tech. Japan, 12 (2014), pp. Tb_19-Tb_24.

3) Koizumi, H., Komurasaki, K., and Arakawa, Y.: Development of the Miniature Ion Propulsion System for $50 \mathrm{~kg}$ Small Spacecraft, Proceedings of the 48th AIAA/ASME/SAE/ASEE Joint Propulsion Conference \& Exhibit, Atlanta, Georgia, USA, 2012, AIAA-2012-3949.

4) Koizumi, H., Inagaki, T., Naoi, T., Kasagi, Y., Komurasaki, K., Aoyama, J., and Yamaguchi, K.: Development of the Miniature Ion Propulsion System: MIPS for the 50-kg-Nano-satellite HODOYOSHI-4, Proceedings of the Space Propulsion, Cologne, Germany, 2014.

5) Kuninaka, H., Nishiyama, K., Funaki, I., Yamada, T., Shimizu, Y., and Kawaguchi, J.: Powered Flight of Electron Cyclotron Resonance Ion Engines on Hayabusa Explorer, J. Propul. Power, 23 (2007), pp. 544-551.

6) Kuninaka, H. and Satori, S.: Development and Demonstration of a Cathodeless Electron Cyclotron Resonance Ion Thruster, J. Propul. Power, 14 (1998), pp. 1022-1026.

7) Koizumi, H. and Kuninaka, H.: Miniature Microwave Discharge Ion Thruster Driven by 1 Watt Microwave Power, J. Propul. Power, 26 (2010), pp. 601-604.

8) Takao, Y., Sakamoto, M., Eriguchi, K., and Ono, K.: Investigation of Plasma Characteristics and Ion Beam Extraction for a Micro RF Ion Thruster, Trans. JSASS Aerospace Tech. Japan, 12 (2014), pp. $\mathrm{Pb} \_13-\mathrm{Pb} \_18$.

9) Takao, Y., Koizumi, H., Komurasaki, K., Eriguchi, K., and Ono, K.: Three-dimensional particle-in-cell simulation of a miniature plasma source for a microwave discharge ion thruster, Plasma Sources Sci. Technol., 23 (2014), pp. 064004-1-11.

10) Takao, Y., Eriguchi, K., and Ono, K.: Effect of capacitive coupling in a miniature inductively coupled plasma source, J. Appl. Phys., 112 (2012), pp. 093306-1-10.

11) Takao, Y., Kusaba, N., Eriguchi, K., and Ono, K.: Two-dimensional particle-in-cell Monte Carlo simulation of a miniature inductively coupled plasma source, J. Appl. Phys., 108 (2010), pp. 093309-1-8.

12) Surendra, M. and Graves, D. B.: Particle simulations of radio-frequency glow discharges, IEEE Trans. Plasma Sci., 19 (1991), pp. 144-157. 